\title{
A circular microchannel integrated with embedded sprial electrodes used for fluid transportation ${ }^{\text {is }}$
}

\author{
Lung-Jieh Yang ${ }^{\mathrm{a}, *}$, Jiun-Min Wang ${ }^{\mathrm{a}}$, Kai-Chung Ko ${ }^{\mathrm{a}}$, Wen-Pin Shih ${ }^{\mathrm{b}}$, Ching-Liang Dai ${ }^{\mathrm{c}}$ \\ ${ }^{a}$ Department of Mechanical and Electro-Mechanical Engineering, Tamkang University, Tamsui 25137, Taiwan, R.O.C. \\ ${ }^{\mathrm{b}}$ Department of Mechanical Engineering, National Taiwan University, Taipei 106, Taiwan, R.O.C. \\ ${ }^{\mathrm{c}}$ Department of Mechanical Engineering, National Chung Hsing University, Taichung 402, Taiwan, R.O.C.
}

Received 28 July 2006; received in revised form 7 October 2006; accepted 13 October 2006

Available online 28 November 2006

\begin{abstract}
This paper proposes a novel method to fabricate a circular microchannel with embedded spiral electrodes used for fluid transportation by inserting glass capillary as sacrificial mould. Utilizing a rolling exposure technique in the photolithography process, the continuous spiral electrode has been formed on the non-planar surface of a glass capillary successfully. Moreover, using the concept of sacrificial layer, the spiral electrodes are transferred on the inner surface of a SU-8 circular microchannel with a diameter of $350 \mu \mathrm{m}$. The fabricated device was regarded as an electrohydrodynamic (EHD) micropump, and has a pumping capacity of $4.5 \mu \mathrm{l} / \mathrm{min}$ herein.
\end{abstract}

(C) 2006 Published by Elsevier B.V.

Keywords: Circular microchannel; Spiral electrodes; Rolling exposure; Electrohydrodynamic (EHD)

\section{Introduction}

As many MEMS or micro fabrication technologies are originally based on the planar process, the three-dimensional micro/nano machining as well as the non-planar micro/nano structures attract more and more attention in the development of novel micro/nano technologies (MNT). For meeting the requirement of providing key components for coils, inductors, solenoids, transformers, mechanical springs or driving electrodes of fluidic pumps in the micro/nano scale, many novel techniques of fabricating three-dimension spiral structures have been proposed accordingly.

The previous works about fabricating spiral features or microstructures can be categorized into the bottom-up and the top-down approaches. For the bottom-up approach, examples of the spiral nanostructures could be synthesized from different constructing blocks such as carbon nanotubes [1] or zinc oxide nanobelts [2]. For the top-down approach, many techniques were also developed by different working principles respectively. In

\footnotetext{
is This paper is presented at the 3rd Asia-Pacific Conference on Transducers and Micro/Nanotechnology (APCOT 2006), held in Singapore, June 25-28, 2006, and is an expansion of the abstract as printed in the Technical Digest.

* Corresponding author. Tel.: +886 932 159193; fax: +886 26209750.

E-mail address: Ljyang@mail.tku.edu.tw (L.-J. Yang).
}

2003, the spiral structures made by focused-ion beam chemical vapor deposition have been presented [3]. Additionally, the spiral structures have been formed by strained SiGe thin film [4] and by the technique of localized stress-introducing technique [5].

In this paper, a top-down approach of fabricating spiral structures is developed. The technique is based on the non-planer photolithography on the thin-film tube [6]. By utilizing this technique, the spiral electrodes are formed on the non-planar surface of a glass capillary successfully at first. Secondly, combining the method of fabricating SU-8 microchannel with circular crosssection in Ref. [7], the authors substitute optical fibers with glass capillaries, which have the spiral electrodes patterned in advance, as the sacrificial mould to implement the embedded SU-8 circular microchannels. Herein, the spiral electrodes were aptly mounted on the inner surface of a circular microchannel. Finally, this SU-8 circular microchannel integrated with couples of the spiral electrodes can be regarded and applied as an electrohydrodynamic (EHD) micropump [8] for fluid transportation.

\section{Device fabrication}

\subsection{Rolling lithography of spiral electrodes}

In order to achieve a structure of spiral electrodes, the technique of rolling exposure should be used. Fig. 1 shows the 


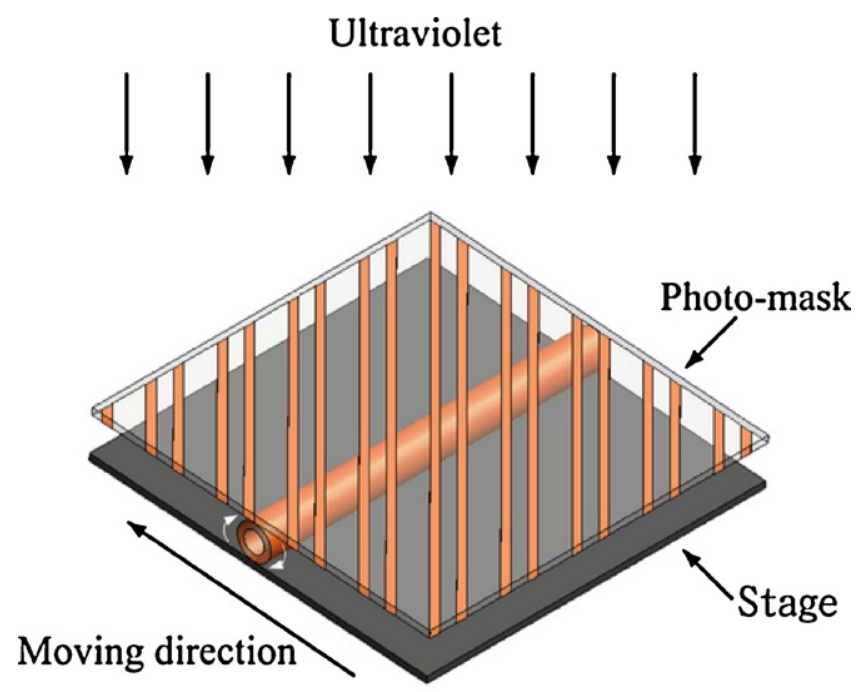

Fig. 1. The working principle of the rolling exposure process on a glass capillary with respect to the stationary photo-mask, the translational stage induces the rolling of the capillary during the UV exposure.

fabrication concept of the spiral electrodes. The glass capillary always contacts closely with the photo-mask and the translation stage at the same time. When the stage is controlled moving, the glass capillary starts to rotate if no slip occurs during the operation. After a complete round of the rolling motion with the continuous ultraviolet (UV) exposure on the glass capillary having photoresist coated in advance, the spiral patterns are transferred from the planer photo-mask to the curved surface of the glass capillary.

A tailor-made rolling module augmented on a conventional mask aligner (Kyowa Riken K-309) is used for the purpose of achieving the above fabrication concept. As the design shown in Fig. 2, the $x$-direction knob of the mask aligner controlling the translation stage connects the stepping motor by a rubber belt. In other words, the stepping motor can control the position and the moving speed of the translation stage. By using this augmented module, the resolution of the resist-pattern on the glass capillary can be achieved to be $40 \mu \mathrm{m}$. The mask aligner

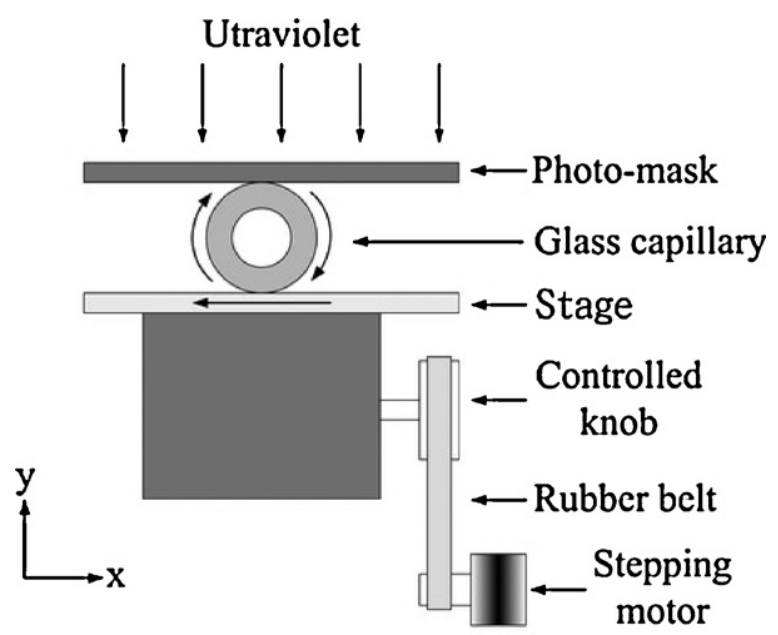

Fig. 2. The design of the rolling exposure module for glass capillaries.

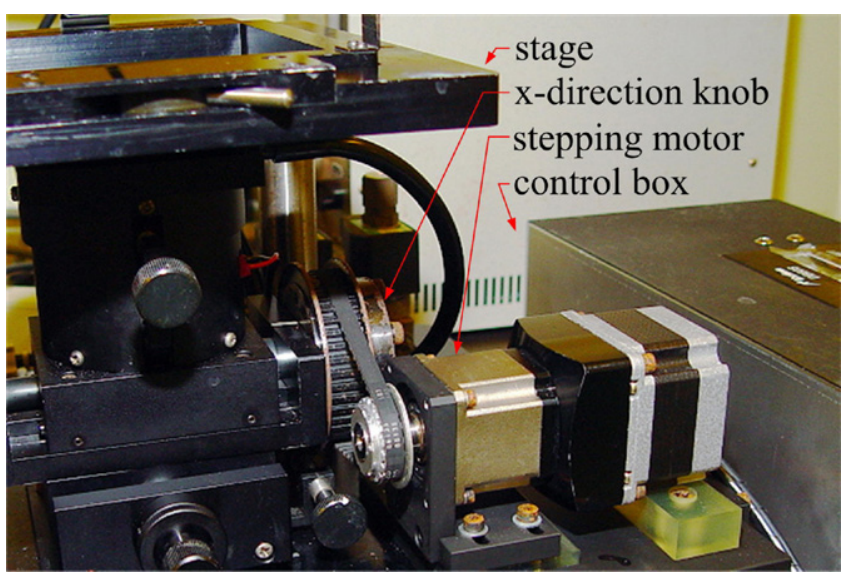

Fig. 3. The mask aligner with the rolling module (include stepping motor and control box).

with a rolling module after assembly and installation is shown in Fig. 3.

For obtaining the spiral pattern of photoresist, the proper layout design of the photo-mask is important. By using a suitable design after rolling exposing of 360 degrees (a full round), the line pattern on the photomask can be transferred to the spiral pattern on the glass capillary successfully. Fig. 4 shows the photo-mask layout design for a single pair of spiral electrode with a gap of $d_{2}$ and a width of $d_{1}$. While performing a complete round, the right end of electrode wires should match to the left ones in Fig. 4. The slant angle of spiral electrodes can be expressed as Eq. (1).

$\tan \theta=\frac{w_{4}}{\pi D}$

$\theta$ is the slant angle of spiral electrodes; $D$ is the diameter of glass capillary; $w_{4}$ is the pitch length for the spiral electrode. Due to the final application as EHD micropumps in this paper, there are two spiral electrodes (a single pair) on the surface of glass capillary. The relation of pitch length $w_{4}$, electrode width $d_{1}$ and electrode gap $d_{2}$ formulated as Eqs. (2) and (3).

$w_{i}, \cos \theta=d_{i} ; \quad i=1-3$

$w_{4}=2 w_{1}+w_{2}+w_{3}$

The dimension control of the electrode parameters mentioned above is very crucial. Either the unsuitable photo-mask design or the improper manipulation during the rolling lithography will result in the incoherent spiral patterns.

Fig. 5 is the illustration of the fabrication process of spiral electrodes on the surface of a glass capillary. Fig. 6 shows the fabricated spiral electrodes. The finest line-width of the spiral electrodes can be achieved as $40 \mu \mathrm{m}$ in this work. The detailed description is shown as follows.

(a) Ti/Au electrical seeding layer is deposited on the cylindrical surface of the glass capillary of $350 \mu \mathrm{m}$-diameter by an electron-beam evaporator. It takes three times of e-beam evaporation to cover the cylinder surface of the glass capillary exactly with $\mathrm{Ti} / \mathrm{Au}$ seeding layer. 
(a)
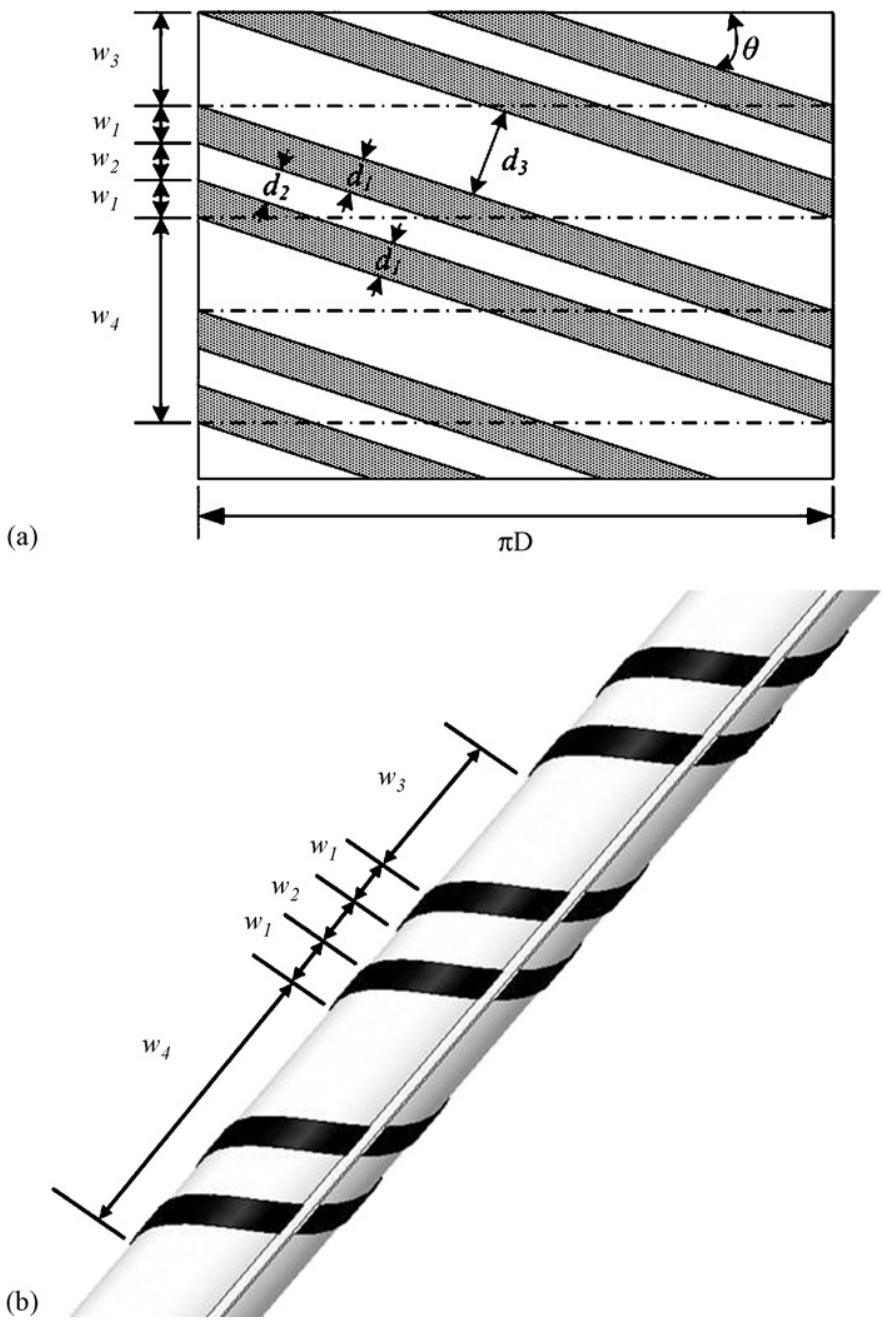

Fig. 4. The design parameters and denotation symbols of the photo-mask used for the rolling lithography; (a) the planer photo-mask; (b) the spiral patterns on the glass capillary.

(b) We coat a photoresist, S1813, on the glass capillary and control well on the dosage of UV light during rolling exposure process by adjusting proper rotation speed of the glass capillary. Therefore, the photoresist of a spiral pattern appears after the developing process.

(c) Buffered oxide etch (BOE) and Au etchant $\left(\mathrm{I}_{2}: \mathrm{KI}\right.$ :water $=$ 1:4:40) are used to pattern the $\mathrm{Ti}$ and $\mathrm{Au}$, respec-
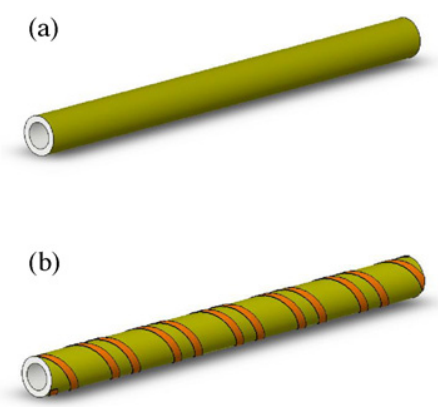

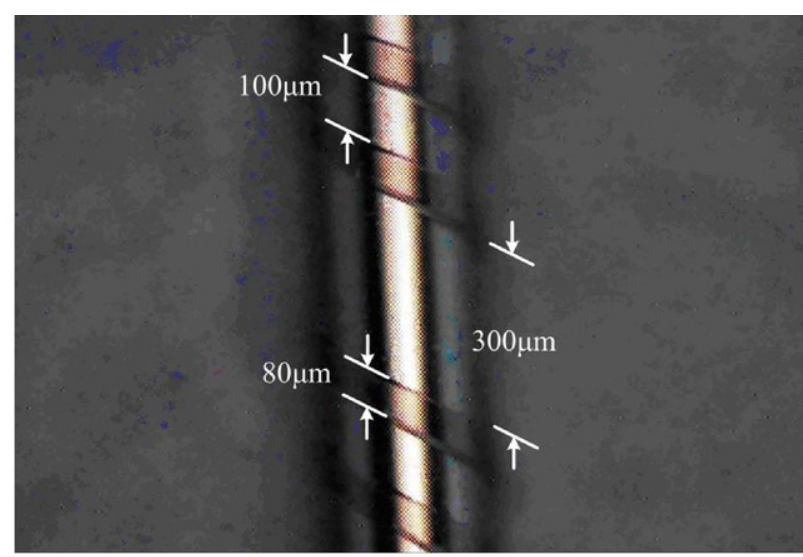

(b)

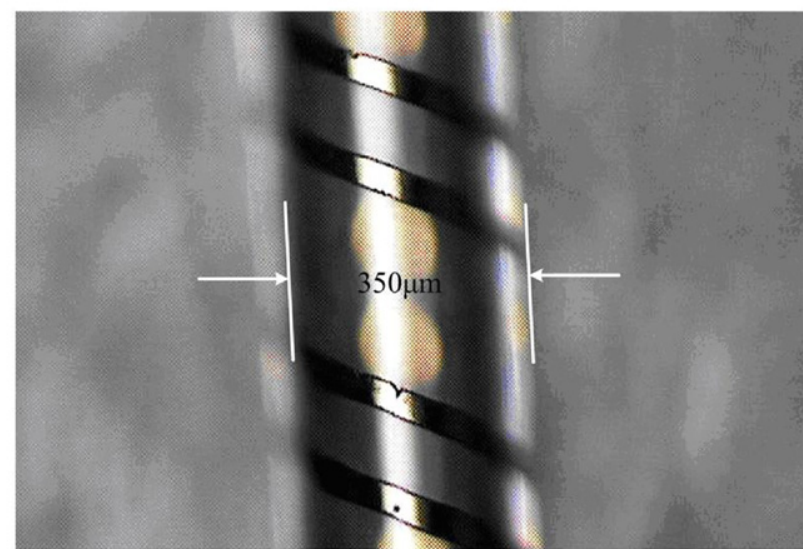

Fig. 6. The fabricated spiral electrodes: (a) the photoresist with spiral patterns on the glass capillary; (b) after electroforming, the spiral metal electrodes are formed on the surface of the glass capillary.

tively, and the seeding layer of spiral electrodes are obtained.

(d) Finally, a $5 \mu \mathrm{m}$-thick nickel was selectively electroformed in order to intensify the structure strength of the spiral electrodes.

\subsection{Fabrication of a circular microchannel with embedded spiral electrodes}

After finishing the process of rolling lithography, the spiral electrodes on the glass capillary can be transferred to the inner

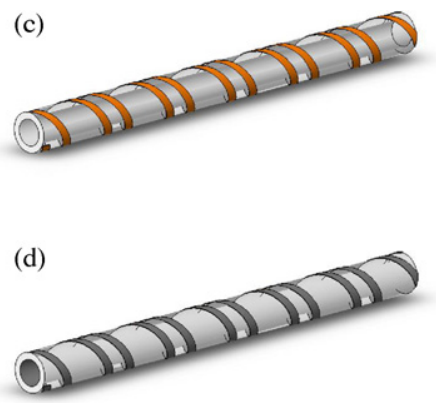

Fig. 5. The fabrication process of spiral electrodes on the surface of a glass capillary; (a) Ti/Au seeding layer is deposited on the surface of capillary by e-beam evaporator; (b) using rolling exposure process to obtain the spiral pattern of S1813 photoresist; (c) the spiral electrodes of Ti/Au seeding layer is patterned by BOE and Au etchant, respectively; (d) a $5 \mu \mathrm{m}$ thick nickel was selectively electroformed on the seeding layer. 
(a)

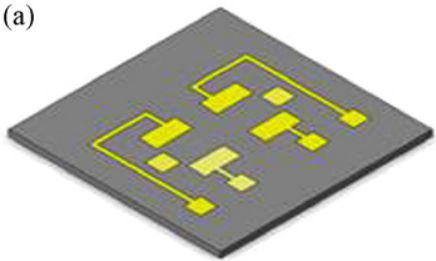

(c)

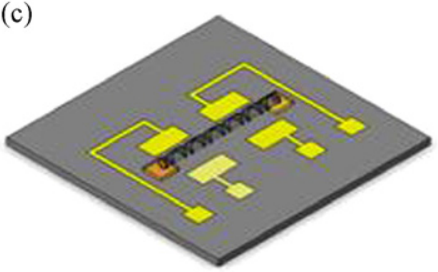

(b)
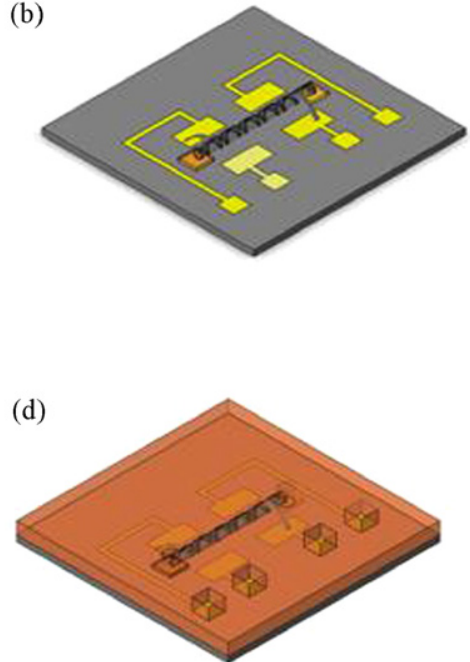

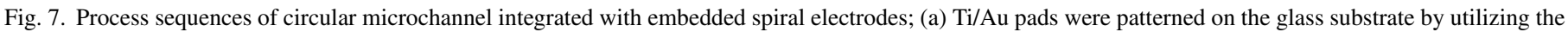

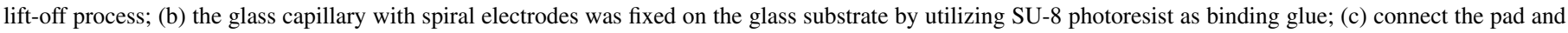

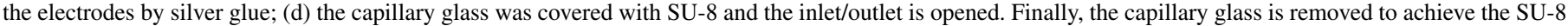
circular microchannel integrated with embedded spiral electrodes.

surface of a SU-8 circular microchannel by regarding the glass capillary as the sacrificial material. Fig. 7 shows the process flow.

(a) A glass substrate was cleaned, and then, evaporated with $\mathrm{Ti} / \mathrm{Au}$ pads by the lift-off process.

(b) The glass capillary with spiral electrodes was fixed on the glass substrate by utilizing SU-8 photoresist as binding glue.

(c) We connect the pads with the input/output electrodes by silver glue.

(d) The capillary glass was wholly covered with SU-8 resist. After patterning the inlet/outlet and etching the capillary glass, the circular microchannel integrated with embedded spiral electrodes can be achieved.

\section{Results and discussion}

\subsection{Sacrificial etching}

The removal of sacrificial layer spends several hours in the previous work of circular microchannel process [7]. In this work, a glass capillary instead of optical fibers is chosen as the sacrificial material. It is for the reason that the capillary force between the sacrificial-layer etchant (HF) and the inner sidewall of glass capillary provides a driving force to pump the etchant into glass capillary automatically.

The etching process is divided into two stages. In the first twenty minutes of etching process, the glass capillary exposed to the entrance window was etched fully by HF acid. Then, the capillarity could drive HF acid from the entrance window to the inner wall of glass capillary. Thirty minutes later, the capillary glass in the microchannel was removed exactly. In summary, it takes only $50 \mathrm{~min}$ to complete the sacrificial etching herein, and the etching time is much shorter than the previous work. The fabricated device of a SU-8 circular microchannel integrated with embedded spiral electrodes is shown in Fig. 8.

\subsection{Application to EHD pumping}

Finally, this device can be regarded as an EHD micropump to drive the ethyl alcohol. It provides not only the driving electrodes available for the pushing of nonpolar fluids, but also constructs a three-dimensional electric field effect (the electric field effect of Ref. [8] is only obvious near the bottom surface of the microchannel surface). It used a CCD image-capturing module to record the EHD pumping velocity of the moving front of gas bubbles in the ethyl alcohol channel-flow through an optical microscope. Fig. 9 shows the continuous, moving images of the EHD pumping flow. The experimental result of the ethyl alcohol pumping rate is $4.5 \mu \mathrm{l} / \mathrm{min}$ by applying a DC driving voltage of $25 \mathrm{~V}$. Such a pumping volumetric rate is genuinely better than the planar type EHD pump [8] with one order of mag-

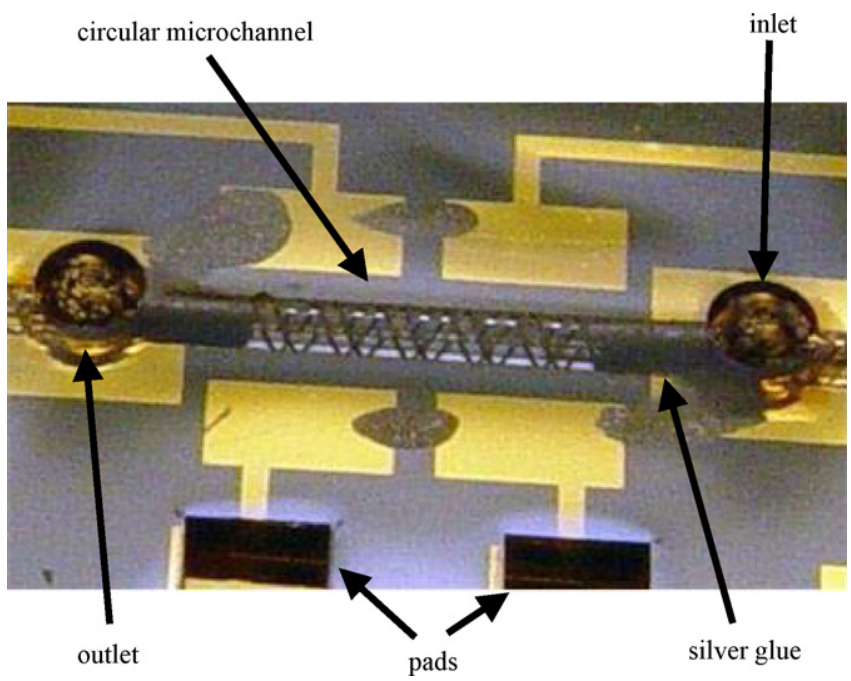

Fig. 8. The device of a circular microchannel integrated with embedded spiral electrodes. 

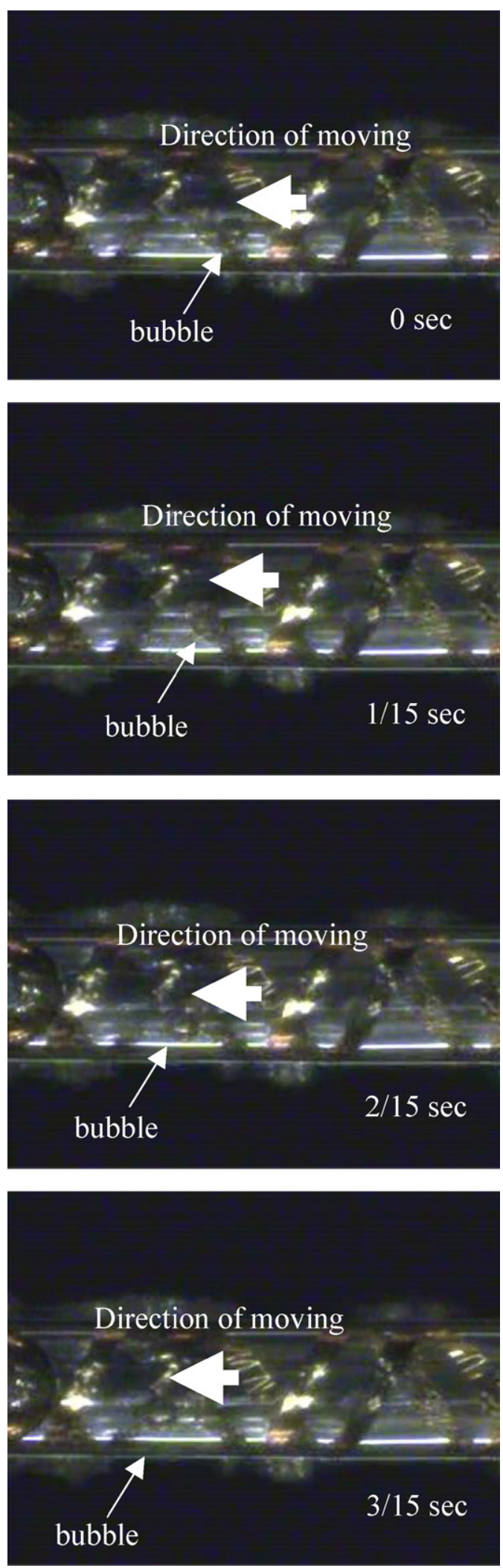

Fig. 9. The four continuous captured images of the spiral electrodes pumping flow (with ethyl alcohol as the working fluid); the moving speed of the bubble is observed to be $785 \mu \mathrm{m} / \mathrm{s}$. nitude. However, the bubble generation due to water electrolysis and the electrode aging issue are still serious and inevitable [8]. As a matter of fact, the spiral electrodes only last for $20 \mathrm{~min}$ and begin detaching from the inner wall of the prototype device.

\subsection{Issue of electrode failure}

How to prolong the lifetime of the working electrodes for EHD micropumps is a difficult problem and has not yet been solved. In this paper the detaching of the spiral electrodes from the inner wall of SU-8 circular microchannel detrimentally limits the continuous working time of no more than $20 \mathrm{~min}$. Two possible reasons as well as their corresponding modifications are addressed in the following.

First, the compressive residual stress in the electroplated nickel layer enhances the detachment of the spiral electrodes from the device. In the fabrication stage after defining and electroplating the spiral electrodes on the surface of glass capillaries (Figs. 5 and 6), no delamination of the spiral electrodes have been observed. Then we believed that the compressive residual stress in the electroplated nickel beneficially helps the electrodes to "clamp" the glass capillary firmly. However, after removing the glass capillary in Fig. 7(a), we have no supporting base available for the spiral electrodes to "grasp". Therefore, it takes for granted that the almost "released" spiral electrodes could be easily flushed away if other external forces applied on. We recommend adjusting the electroplating condition of nickel layer to have its residual stress as small as possible [9].

Second, the intrinsic poor adhesion between the SU-8 polymer surface (of the circular microchannel) and the metal electrodes could not guarantee the effective immobilization of the spiral electrodes in the device. Furthermore, the thermal expansion coefficient of SU-8 and nickel are quite different. Any apparent temperature rise during the EHD operation will cause serious thermal stress to destroy the poor adhesion of the spiral electrodes. Suitable polymer protection (e.g. using parylene [10],) on the edge of spiral electrodes may provide the mechanical "clamping" structures to resist the thermal shock or even the residual stress of electroplated nickel mention previously.

\subsection{Other problems of fabrication}

Besides the unsuitable photo-mask layout, the slip of the glass capillary when performing the rolling exposure process will also cause the mismatch pattern of the spiral electrode. It is due to the different frictions for the touch points on the top and the bottom of the glass capillary respectively. It could use a piece of glass or other plate on the wafer chuck to adjust the bottom friction of the glass capillary. In addition, the Ti/Au seeding layer is evaporated on the cylinder surface of the glass capillary by taking three times of e-beam evaporation which would sometimes make the etching $\mathrm{Ti} / \mathrm{Au}$ difficult (some unspecified portions of metal are hard to remove). There is a new evaporator with rolling chuck in Ref. [6] could improve the time-consuming, repetitive evaporation on the surface of glass capillary in this paper. 


\section{Conclusions}

A new fabrication of spiral electrodes is presented in this paper. Utilizing the approach of rolling exposure and photolithography procedure, the continuous spiral electrodes were formed on the surface of a circular glass capillary with $350 \mu \mathrm{m}$ diameter. The finest line-width of the spiral electrodes is $40 \mu \mathrm{m}$. Moreover, the spiral electrodes were integrated with a microchannel. Upon the HF etching process, etching time could be shortened to fifty minutes only, due to the mechanism of automatic capillary suction, and the spiral electrodes are embedded into the microchannel. Finally, it could act as an EHD micropump and drive the ethyl alcohol to have the maximum volumetric flow rate of $4.5 \mu \mathrm{l} / \mathrm{min}$ by applying a DC driving voltage of $25 \mathrm{~V}$.

\section{Acknowledgements}

The authors appreciate the financial support of the WHAM-BioS project (94-EC-17-A-05-SI-017) from Ministry of Economic Affairs, Taiwan; and of the project (NCS-94-2212E032-013) from National Science Council, Taiwan.

\section{References}

[1] J.P. Cheng, X. Zhang, J. Tu, X. Tao, Y. Ye, F. Liu, Catalytic chemical vapor deposition synthesis of helical carbon nanotubes and triple helices carbon nanostructure, Mater. Chem. Phys. 95 (2006) 12-15.

[2] X.Y. Kong, Z.L. Wang, Spontaneous polarization induced growth of $\mathrm{ZnO}$ nanostructures, Proc. Int. Conf. Solid-State Integr. Circuits Technol. 2 (2004) 894-897.

[3] S. Matsui, Three-dimensional nanostructure fabrication by focus-ion-beam chemical vapor deposition, Proc. International Conference on Solid-State Sensors, Actuators and Microsystems (Transducers'03) vol. 1, 2003, pp. 179-181.

[4] D.J. Bell, Y. Sun, L. Zhang, L.X. Dong, B.J. Nelson, D. Grützmacher, Three-dimensional nanosprings for electromechanical sensors, Proc. International Conference on Solid-State Sensors, Actuators and Microsystems (Traansducers'05) vol. 1, 2005, pp. 15-18.

[5] L. Xia, W. Wu, J. Xu, Y. Hao, Y. Wang, 3D nanohelix fabrication and 3D nanometer assembly by focused ion beam stress-introducing technique, Fourteenth IEEE International Conference on Micro Electro Mechanical Systems (MEMS’06), 2006, pp. 118-121.
[6] T. Mineta, M. Abe, H. Kubo, E. Makino, T. Shibata, Fabrication of 3D micro structures from evaporated thin film tube, Proceedings of ICEE/APCOT'04, 2004, pp. 322-325.

[7] L.-J. Yang, Y.-T. Chen, S.-W. Kang, Y.-C. Wang, Fabrication of SU-8 embedded microchannels with circular cross-section, Int. J. Mach. Tools Manuf. 44 (2004) 1109-1114.

[8] L.-J. Yang, J.-M. Wang, Y.-L. Huang, The micro ion drag pump using indium-tin-oxide (ITO) electrodes to resist aging, Sens. Actuators A: Phys. 111 (2004) 118-122.

[9] V. Holpuch, J. Vítek, Electrodeposition of nickel from sulphosalicylate solutions, Surf. Technol. 5 (1997) 89-96.

[10] J.M. Wang, L.J. Yang, Electrohydrodynamic (EHD) micropumps with electrode protection by parylene and gelatin, Tamkang J. Sci. Eng. 8 (3) (2005) 231-236.

\section{Biographies}

Lung-Jieh Yang received his MS degree from Tamkang University, Taiwan in 1991 and PhD degree from the Institute of Applied Mechanics, National Taiwan University, Taiwan in 1997. He is currently a professor of the Department of Mechanical \& Electro-mechanical Engineering and the director of Instrument \& Experiment Center, Tamkang University, Taiwan. His current research interests include micro-fluidics, lab-on-a-chips and polymer MEMS technologies.

Jiun-Min Wang was born in 1978 in Taipei, Taiwan. He received his MS degree from the Department of Mechanical \& Electro-mechanical Engineering, Tamkang University, Taiwan, in 2003. He is now working toward his $\mathrm{PhD}$ degree in Tamkang University, Taiwan.

Kai-Chung Ko was born in 1980 in Taichung, Taiwan. He received his BS degree in the Department of Mechanical \& Electro-mechanical Engineering, Tamkang University, Taiwan, in 2000. He is now working toward his MS degree in Tamkang University, Taiwan.

Wen-Pin Shih received his BS degree from National Taiwan University in 1997, and $\mathrm{PhD}$ degree from Cornell University in 2004. He is currently an assistant professor of the Department of Mechanical Engineering, Nation Taiwan University. His research interests are micromechanics and dynamics related to MEMS, nanomanufacturing, biosensors and nanoactuators.

Ching-Liang Dai received the MS degree in the applied mechanics from National Taiwan University, Taiwan, in 1993, and the $\mathrm{PhD}$ degree in the mechanical engineering from Nation Taiwan University, in 1997. He is currently an associate professor at the Department of Mechanical Engineering, National Chung Hsing University, Taiwan. His research interests are CMOS-MEMS, integrated microsensors and RF MEMS. 